\title{
Ongoing processes of managing consent: the empirical ethics of using video- recording in clinical practice and research
}

\begin{abstract}
Using video to facilitate data collection has become increasingly common in health research. Using video in research, however, does raise additional ethical concerns. In this paper we utilise family therapy data to provide empirical evidence of how recording equipment is treated. We show that families made a distinction between what was observed through the video by the reflecting team and what was being recorded onto videotape. We show that all parties actively negotiated what should and should not go 'on the record' with particular attention to sensitive topics and the responsibility of the therapist. Our findings have important implications for both clinical professionals and researchers using video data. We maintain that informed consent should be an ongoing process and with this in mind we present some arguments pertaining to the current debates in this field of health care practice.
\end{abstract}

Key words: Informed consent, video, qualitative, discourse, recording

\section{Introduction}

The drive towards evidence-based practice and the need to utilise research at strategic and operational levels ${ }^{1}$ has meant a development in methodological strategies in this discipline. The use of video for research and clinical reflection has become progressively more popular for improving practice and advancing our understanding of patient care ${ }^{2}$. For practitioners, video data facilitates reflection on these complex challenges and allows them to consider the impact of their practice on their patients ${ }^{3}$. For qualitative researchers, video offers diversity in the presentation of findings and allows for the creation of clips, still frames and transcribed text ${ }^{4}$. 
Health care settings are one location whereby the mechanics of social interaction can be systematically scrutinised through the use of video ${ }^{5}$. In this way the analyst is able to identify possible antecedents and consequences of interactional patterns ${ }^{6}$. Using video in health settings, however, can present ethical challenges and heighten sensitivity to participant protection. The issue of patient privacy and the legal burden of publicising evidence can make research ethics committees hesitant to grant approval ${ }^{7}$. One of the core concerns for ethics committees are the additional questions invoked regarding informed consent ${ }^{8}$. In particular, the use of video in qualitative health research has fuelled fears related to data protection and revelations of identity; because in a range of settings (including clinical ones) the creation of a semi-permanent record adds an additional concern for participants ${ }^{9}$. The existence of a video record increases the possibility for third parties to access its content which may cause embarrassment or emotional distress to participants. Significant attention has been paid to the content of consent forms and the accompanied participant information sheet but recently there has been growing attention paid to the process of consent procedures $^{10}$.

One area of health care that commonly utilises video recordings for reflection, training and research purposes is family therapy. The systemic approach to family therapy aims to treat families and directs clients to concentrate on a relational process and is a language based way of working therapeutically ${ }^{11}$. The practice of using video recordings as a clinical tool allows for reflective learning through observation and feedback ${ }^{12}$ thus presenting an opportunity to see patterns and work collaboratively with other professionals ${ }^{13}$. By utilising video data collection and allowing practitioners to reflect upon empirical data provides a forum for discussions and improvements rather than reducing it to abstracted models or case studies ${ }^{14}$. 
Thus video provides a mechanism through which practitioners have an immediate source of clinical feedback through which they can reflect on the emergent quality of the process of therapy as it is embodied by the therapist ${ }^{2}$. In addition to reflective practice, the clinical governance agenda has promoted the development of evidence-based practice and family therapy is one area of health care that uses video research more formally to evaluate practice $^{15}$.

\section{Consent for research and training}

To video record family therapy sessions, the therapist must acquire consent from family members. For the purposes of research the requirement for informed consent is more stringent and regulated than it is for clinical reflection, due to the Department of Health regulatory framework for research. This process of informed consent is an expression of trust and it is through this process that participants express confidence in the professionals to manage potential risk ${ }^{1}$. The basis of informed consent, therefore, is that participants are given adequate information about the research in order for their autonomy to be respected, and they trust that the promises made will be implemented. The professional has responsibility for ensuring that this trust is founded, that the participant has capacity to provide informed consent, has done so voluntarily and that withdrawal rights are clarified.

A common argument against the use of video recordings for research has been that the presence of the video camera unduly influences the behaviour of participants, but although participants may display occasional awareness of the recording devices this is not automatically a hindrance ${ }^{16}$. Furthermore, although participants may initially be uncomfortable with the presence of a video camera, over time people adjust and become acclimatised to its presence ${ }^{12}$. While the acclimatisation is beneficial in research terms, there 
are ethical implications for the consent process. Participant recall of consent taken at the start of therapy, for example, may consciously diminish over the course of a number of therapy sessions. Ethically, therefore, the revisiting of consent should be welcomed throughout the course of therapy. In this way, rather than simply being an episodic activity, consent is an iterative, ongoing process.

\section{Aims of the paper}

1. Through analysis we will show that the long-standing ethical issues for both clinical care and research, such as informed consent, confidentiality, data protection and avoiding foreseeable harm to participants as related to the four core principles of autonomy, justice, beneficence and non-maleficence ${ }^{17}$ cannot be treated in isolation as an a priori concern but are negotiated by members in the interaction

2. We will demonstrate that informed consent in relation to the recording equipment is an iterative process and should be addressed throughout therapy sessions and revisited through a process of negotiation between participant and therapist of what goes on or off the record.

3. These key ethical issues of consent, confidentiality and so forth require a foundation of trust and responsibility which are treated by the participants as fundamental concerns

The main aim of this paper, therefore, is to provide an empirically grounded account that utilises actual participants of therapy to provide some transparency on the debate regarding the ethics of video recording for research and the arguments around informed consent. 


\section{Methods}

This paper utilises a qualitative, discourse analytic approach to explore the important ethical issue of using recording equipment for health research.

\section{The therapeutic setting}

The data for this paper was provided by a systemic family therapy team based in the United Kingdom. For the purpose of this analysis it is not our concern to debate the effectiveness of different approaches, rather we take a meta-perspective of the more general issues relating to gaining informed consent for researching clinical practice. For this paper we were provided with video-taped data of family therapy sessions. In the setting of family therapy it is fairly typical for the sessions to be video-recorded as part of normal clinical practice, as a reference point for therapists. It is, however, less usual for the video tapes to be used for research and this therefore requires additional consent and sensitivity. This is because the video tapes are taken away from the clinical site to an academic one, and the participant's voices are used in dissemination, thus raising additional risk of identification.

For this study, the data are provided by two therapists who took consent from four families with the pseudonyms of the Clamp family, the Bremner family, the Niles family and the Webber family (see table one). Specific consent was obtained to use the video for research purposes. The video-tapes total approximately 22 hours of family therapy. In keeping with the discursive epistemology, sampling is appropriate.

\section{The approach}

There are a number of useful qualitative approaches available for studying family therapy and discourse analysis is particularly methodologically congruent with family therapy theory and 
practice $^{18}$. It thus makes intuitive sense to use a language-based methodology to explore one of the talking therapies. For our research a number of insights from discourse analysis and from conversation analysis are applied in drawing out pertinent features in the transcribed data ${ }^{19}$. This form of discourse analysis has a commitment to study talk in social practice ${ }^{20}$ and enables the researcher to explore the contribution of all parties within the therapy from their respective positions ${ }^{15}$. This allows for a rigorous and empirically grounded analysis of the data. For this research we utilised the standardised Jefferson transcription system ${ }^{21}$. This type of transcription process, as compared with other methods, makes the data available for critical scrutiny in the academic community.

\section{Ethics}

For this study the clinical manager of a family therapy centre was initially approached for permission to consult the family therapy team. Discussions with family therapists subsequently took place and their cooperation and consent obtained. It was agreed at this point that the therapists would take responsibility for acquiring consent from families. Therapists took consent from adult family members, for themselves and on behalf of their children. While the complexities surrounding the competence of children to provide assent to participate in research is acknowledged, ethical guidance regarding actively obtaining assent from children has become more sophisticated since data were collected. Videotaping for training and research purposes was routinely collected but consent for conducting this particular project was additionally agreed between the therapist and parents. The principlist approach to ethics ${ }^{22}$ was utilised for this research, the four core principles of which are autonomy, justice, beneficence and non-maleficence. 


\section{Analysis}

In -depth analysis revealed that there were four key issues relating to the recording equipment that were brought into focus during the dialogue.

1. The therapist assumes responsibility for managing the ongoing consent process.

2. Participants make a distinction between the reflecting team listening behind the video camera and the equipment actually recording what is said

3. Participants actively negotiate what should go on or off the record

4. Participants draw particular attention to the introduction of sensitive topics as places where the recording equipment becomes more relevant

Theme 1: Responsibility

Throughout the therapy session, the therapist takes steps to emphasise his role as facilitator in the ongoing consent process.

\section{Extract 1: Webber family}

1 FT: I'll leave this here if you want to have a read through it and if it's okay (.) to sign it (.) the bottom bit is for me $t^{\prime}$ sign just to say I will look after the $\downarrow$ tape

Extract 2: Niles family

$1 \mathrm{FT}$ :

will you sign that one as well and then I sign the bottom to say (0.6) I won't put it on ITN news or anything like that

3 Dad: Fair enough (.) she's a proper er (.) news freak she is

Prior to commencing the first therapy session it is the therapist's professional and moral duty , having explained the nature of the project to the families, to obtain their written informed 
consent with a view to covering eventualities that might arise in the current and subsequent therapy sessions. In the above extracts, he points out the potential anxiety families may harbour about the tapes by stressing his role of caretaker. In extract one, he informs the family that he will 'look after the tape' (line 2) and in extract two he uses humour to convey the potential extremes and shows that he will not do this 'I won't put it on ITN news' (line 2)

16. While all parties have an opportunity to make reference to the video in relation to ongoing consent, ultimately it is the therapist who has an ethical obligation to safeguard the participants. Despite considerable attention being paid to the requirement to take consent at the beginning of the first therapy session, less attention has been given to the ongoing process of consent throughout a series of therapy sessions. The responsibility of the therapist, therefore, extends beyond the first session. Furthermore clarification of both the active and passive roles of the camera is necessary. This should form part of the families' initial basis for providing consent although it is likely to be necessary for the therapist to remind families at intervals throughout.

\section{Theme 2: Recording or listening?}

It is noteworthy here, as is borne out by the data, that family members make a distinction between the camera which has a function not only as a portal device to the reflecting team but also as a recording device that provides a record of what is being said.

\section{Extract 4: Clamp family} watching on a T.V monitor in the other room

The recording device has two functions in family therapy. First it allows the reflecting team to observe and later comment on therapy, and second to record the therapy for training and 
research purposes. Here the therapist introduces the camera as a having a passive role to allow contributions from other clinical professionals, it does however also have the capacity to record the session.

\section{Extract 5: Niles family}

1 Steve: Is the camera on?

2 FT: It is (.) $\uparrow y e s$ it is (.) and the video's $\downarrow$ probably tapin' as well

Part way through the therapy session, the eldest child, Steve, asks the therapist whether the camera is on 'is the camera on?' (line 1).This raises to the possibility that although a priori consent has been provided, the participants still revert to the status of the camera as on or off. Simply the therapist could provide a yes or no response but he chooses to qualify the status of the camera in more detail by differentiating between its active and passive functions. He reports that the camera is on, in response to Steve's question although through the use of 'probably' (line 2) he conveys that he is not certain of the active recording status of the machine.

\section{Extract 6: Clamp family}

$1 \quad \mathrm{FT}$ :

the <video camera> (.) is 个off (.) when I'm not >in the room< (.) okay (.) so no-one will be listenin' or recordin' or anythin'

3 Dad: So $>$ we can talk about ya< when yo(h)u're go(h)ne

The therapist provides further clarification to the families regarding the function of the recording equipment. The therapist clarifies that when the video camera is off, it is definitely not recording and neither will the reflecting team behind it be listening 'no-one will be listening or recording or anything' (line 2). The above extract draws out what is and is not 
listened to by the reflecting team and subsequently what does and does not go on record. The interesting response from the father is couched in humour 'so we can talk about ya when you're gone' (line 3) which seems to point to the practical interactional dimensions of whether the tape machine discourages certain types of topic $\left(\mathrm{cf}^{16}\right)$.

These three extracts emphasize that although the families give consent for recordings at the start of their therapeutic journey, the therapist and families do further intermittent work throughout to revisit consent. At various junctures, distinctions are made between what is being heard by others and what goes on the record. This demonstrates that there are potentially layers of audience, the therapist in the room, the overhearing reflecting team and the researcher. The therapist has an ethical obligation to clarify to family members at different points in the session that the conversation will be listened to by others.

Theme 3: Negotiating what goes on or off the record

Our analysis demonstrates that the focus of what goes on and off the record in therapy and research results from a process of negotiation.

\section{Extract 7: Webber family}

$1 \quad \mathrm{FT}$ : well that's (0.2) yea::h (0.4) l'll go and talk > with the team< hh for a bit $(0.2)$ we'll close down the $\downarrow$ camera an' everythin' (.) so you're in total (0.2) (sp[ace) then

3 Mum: [oh (.) is it on $\downarrow$ then?

$5 \quad \mathrm{FT}$ : Oh 个yeah (.) sorry [I should have said that at the beginning

6 Mum:

[Oh

$7 \quad$ Mum: $\quad$ YYou said it'd be on $>$ didn't you< 
Dad:

((nods))

The therapist refers to the camera being on by his reference to turning it off, which treats the camera being on as unproblematic and already known. The mother, however, treats this as problematic through her questioning, positioning the therapist as accountable. This is noted through the preface to the question and the nature of the question. She responds initially with the discourse marker, 'oh' ${ }^{23}$, used to signal that something has been noticed 'just now' ${ }^{24}$. This functions as a 'change of state' token, indicating something that wasn't known before ${ }^{25}$. By asking if the camera is on, signals that this wasn't known by the mother. The apology in the therapist's response indicates that he also treats this as accountable ${ }^{26}$. What this ongoing negotiation of on/off demonstrates is that the responsibility for the presence and activity of the recording equipment is an ongoing process, rather than a one off event at the start of the first therapy session.

\section{Extract 8: Webber family}

$1 \mathrm{FT}$ :

cuz it sounds like <the two of you > '[ave some respect

2 Dad: [Can you jus' (.) >can you jus' hold it there a

3

minute $<$

4 Mum:

Go on then

5 Dad

个Is that on?

6 Mum:

heh heh heh

$7 \quad \mathrm{FT}$ :

It is yes

8 Dad:

Can you jus' ask 'em to turn it off for one second

$9 \quad \mathrm{FT}$ :

$\operatorname{Er}($.$) yes (.) they should be listenin' so they should jus' press the power switch$

In this extract the father's first attempt to intervene interrupts the therapist's summing up of previous discussions (line 2). At this point the father then makes reference to the camera by 
questioning its status as on or off (line 5). In combination with his subsequent request to turn the camera off (line 8), this functions as a pre-question to the main question by which he implies that he has something to say that he does not want on record.

What this negotiation regarding the requested change of status of the camera from on to off demonstrates is the extent to which participants continuously manage their orientation to the recording equipment. The evidence from this extract suggests that families have difficulty in asserting themselves in requesting a change in recording status. This is evident in the lengthy preliminary interactional work leading to the camera being turned off. This is demonstrated by the father's simple request which results in the recording being temporarily suspended. What this highlights is that family members have some trouble in asking for consent to be revoked, even temporarily. This is particularly notable given that therapists (and researchers) go to significant lengths to reassure families at the start that they have the right to ask. There is a general ethical issue raised by this regarding the control of the recording equipment and who has responsibility for turning it on and off. It is this responsibility that is managed at the time by the families and the therapist. Although it is potentially more difficult for clients to initiate requests to suspend the recording, this may be facilitated by the provision of an exit strategy during the initial consent process. Thus, should the need arise this affords them the opportunity to exercise their autonomy at later points in the therapy.

\section{Theme 4: Introducing sensitive topics}

The emotive nature of the therapy process may mean that the families become absorbed in the content of the session and less actively aware of the presence of the camera. The juxtaposition of the asymmetry between family members and therapist may mean that 
requesting suspension of the recording can be daunting for clients and thus there are occasions where the therapist takes responsibility for the ongoing process.

Extract 9 : Clamp family

$1 \mathrm{FT}$ ...... >you know< there is a team of people (.) there's Hannah today (.) who who's

2

FT:

FT: helping out (.) and that we video the sessions now I know that you've both agreed to that [but I think given what we're talking about $=$

Mum:

[Yeah

$=$ is particularly sensitive if you decide that you don't want these sessions video' $\mathrm{d}$ hh that is fine (.) if you're comfortable with the video then that's

Mum: $\quad \downarrow$ Yeah equally fine (.) but >if at some point $<$ (.) > you know< particularly when Joe joins us (.) >you know< (.) one or all of you think (.) <okay> .hhh we want it switched off now (.) < then that's okay> (.) that doesn't mean we stop >you know< $($.$) we can carry on$

This extract shows that what could be seen as a sensitive topic is co-constructed. Throughout the dialogue, the therapist gives prominence to the topic as 'sensitive' 'what we're talking about is particularly sensitive' ( line 5) as having relevance to the recording equipment and as such, the process of informed consent. What this does is manage the sensitivity on behalf of the family; it is their sensitive issue to be dealt with. The noting of consent at this point (line 2-3), functions as a marker of the sensitive nature of the current topic of conversation. It is usual practice to take consent at the beginning ${ }^{27}$, but this marks a topic transition and highlights the recording equipment as important here. Through his narrative he demonstrates to the family that informed consent for recording is not rigid and functions as a reminder to them that some aspects of the therapy can be 'off the record' without affecting clinical care (8-10). In this extract, ultimate responsibility for the recordings rests with the therapist. 


\section{Discussion}

Initial consent for recording therapy sessions for research purposes involves a delicate process of information giving which needs to be sensitively tailored to individual participants, and obtaining written agreement to participate. Research ethics committees have a distinct role to play in the consent process in order to protect participants and researchers. Our analysis demonstrates that when the therapist takes initial consent for the recordings for research, there is some effort to establish trust through the highlighting of responsibility. The therapist has a professional obligation to reiterate key ethical principles such as confidentiality, data protection and avoiding foreseeable harm, in an accessible language emphasising his duty of care to look after the recordings and protect the families' identities. This is especially important given that there is a possibility with video data that participants are visually identifiable. The process of clinical practice, however, can be unpredictable and thus the initial consent at the start of session one, cannot encompass all eventualities and unanticipated events both within a single therapy session and over the course of several sessions. This raises questions regarding who takes responsibility for managing unanticipated topics/events during the therapy in relation to the continuation of recording. This is more salient if the recording is to transcend a single session and continue longitudinally. Where recordings are used for research purposes ethical considerations regarding consent are common both across longitudinal and cross sectional studies. However, the temporal distance between initial consent in ongoing research means that the obligation to reiterate the agreement of the participants is more profound. 
In this paper we demonstrate that family members, at times of particularly sensitive topic introduction, do take time to initiate revisiting consent and the presence of the recording device. In family therapy, family members are likely to discuss a range of personal and sensitive issues and yet in our corpus of four families across several therapy sessions, we only have one instance (extract 8) in which a family member requests that the camera cease recording. This suggests that family members have to do some interactional work to request suspension of the recording and this highlights the status of the therapist who assumes a position of authority. This is evident in the analysis in the way that requests for suspension are managed by being hedged and mitigated.

The therapist emerges as responsible for managing consent as an ongoing process. As our analysis shows, family therapists are aware of the issues of power that exist in the clinical context and may take initiative in checking with family members that consent is still valid. In therapy, however, all parties are dealing with heightened emotions and the complexity of therapeutic discussions. It is feasible, therefore, that both the therapist and family members may forget the presence of the camera. Additionally there may be an added conflict of interests for the therapist. For example, in health care settings it is not uncommon for the practising health care professional to also be the researcher ${ }^{28}$ or acting on behalf of a colleague researcher. Informed consent is a pre-requisite to help protect participants' fear of adverse consequences on their care ${ }^{27}$ and ensure they are made aware of their right to withdraw at any time, without giving a reason ${ }^{29}$. This highlights some of the complexities of decisions regarding who takes consent, the therapist or the researcher, with additional sensitivities if the therapist has both roles. If the therapist is collecting consent on behalf of a researcher it is essential that the therapist and researcher have effective communication to ensure that the therapist is clear about how the data will be used. If the researcher takes 
consent then those individuals will require training in communicating with families and appreciation of capacity issues.

In the day-to-day practice of research, researchers are faced with numerous subtle and nuanced decisions that require their professional judgement ${ }^{30}$. Our analysis has demonstrated that there are ethical issues to be considered by both therapists and researchers throughout their projects. From our analysis it is evident that the therapist has responsibility to act in the best interests of their clients and work in an ethically correct way. The implementation of the ethical principles is not only the responsibility those collecting the data, but also the researcher analysing and disseminating the findings, thus all parties have an obligation to protect participants.

The negotiation of the process of informed consent for using video in both clinical practice and research raises particular issues regarding responsibility, authority and trust. We have demonstrated that the recording equipment is both a portal to the reflecting team and is also a device which can create a semi-permanent record, for training and research purposes. Even in cases where the focus of recording is more on the therapist for supervisory or training purposes, the same ethical concerns are invoked. Video is a valuable resource for therapists in training ${ }^{31}$ and for researchers. Despite the ethical caveats outlined in this paper the benefits video recording mean that therapists should not be dissuaded from its use.

We therefore make a number of recommendations for both therapists and researchers. First using video for reflection, training and research has a number of benefits and with technological advancements we encourage its use. Second, it is essential to safeguard participants and therefore, the process of consent requires frequent revisiting by the therapist 
throughout both single sessions and longitudinal courses of therapy. Third, therapists and researchers should be aware of the difficulty that family members may have in voicing their anxiety about continued recording at certain points in the therapy. It is helpful therefore, if the therapist has a conscious awareness of this and provides opportunities for families to express their wishes. Fourth, the research and clinical community rely on a strong evidencebase to inform their practice and facilitate their understanding of important issues. Thus it is necessary to increase reporting of ethical processes and practices ${ }^{32}$

\section{Conclusions}

In our paper we have shown that ethicality is a process and it is the members of the interaction in the first instance that manage the unfolding nature of this process. Ethical practice, therefore, involves both researcher and participants in that the researcher should be mindful of his/her obligations and responsibilities in the general conduct of research and the participants' rights and dignity should be protected. The therapist has responsibility for revisiting consent issues regardless of whether they or the researcher took initial consent. By viewing consent as ongoing, members have the opportunity to reconsider their right to withdraw, all or parts of the data. For example our analysis of the negotiation of the status of the recording equipment as on or off, was collaboratively agreed. In instances where the health care professional is also the researcher, additional caution is required as there may be vested interest in promoting compliance. While complete neutrality is an ideal, it can only be achieved if the therapist is reflective of their potential coercive influence. Continuing informed consent and opportunities for withdrawal are not confined to this setting but will be important issues in all health care research. These findings have implications for recording in any health care context where the use of video has become more widespread. With such progress, however, comes the need to afford adequate protection for participants. Researchers 
and practitioners need to be constantly aware of how difficult it may be for participants to suspend or withdraw their consent during an intervention. The theme of what goes on and off the record and when those decisions can be made, will be a familiar dilemma for academics and practitioners from many disciplines. Ethical responsibility should not be conferred solely upon certain individuals, rather, consent issues and, for our paper recording issues should be addressed by all parties involved in clinical practice and research. 


\section{References}

${ }^{1}$ Hansson, S. O. (2006). Uncertainty and the ethics of clinical trials. Theoretical Medicine and Bioethics, 27, 149-167.

${ }^{2}$ ledema, R., Forsyth, R., Georgiou, A., Braithwaite, J., \& Westbrook, J. (2007). Video research in health: visibilising the effects of computerising clinical care. Qualitative Research Journal, 6 (2), 15-30.

${ }^{3}$ ledema, R., Merrick, E., Rajbhandari, D., Gardo, A., Stirling, A., and Herkes, R. (2009). Viewing the taken-for-granted from under a different aspect: A video-based method in pursuit of patient safety. International Journal of Multiple Research Approaches, 3, 290-301

${ }^{4}$ Miles, B. (2006). Moving out of the dark ages: An argument for the use of digital video in social work research. Journal of Technology in Human Services. 24 (2/3), 181-196.

${ }^{5}$ Heath, C. (2004). Analysing face-to-face interaction: Video, the visual and material. In D. Silverman (ED). Qualitative Research: Theory, Method and Practice. Second Edition. (pp: 266-282). London: SAGE.

${ }^{6}$ Bottorff, J. L. (1994). Using videotaped data recordings in qualitative research. In J. M. Morse (Ed). Critical Issues in Qualitative Research Methods. (pp: 244 - 261). London: SAGE Publications.

${ }^{7}$ ledema, R., Long, D., Forsyth, R., \& Lee, B. (2006). Visibilising clinical work: video ethnography in the contemporary hospital. Health Sociology Review, 15, 156-168.

${ }^{8}$ Richards, H. M \& Schwartz, L. J. (2002). Ethics of qualitative research: are there special issues for health services research? Family Practice, 19, 135-139. 
${ }^{9}$ Giordano, J., O’Reilly, M., Taylor, H., \& Dogra, N. (2007). Confidentiality and autonomy: The challenge(s) of offering research participants a choice of disclosing their identity. Qualitative Health Research. 17 (2): 264-275.

10 Johnson-Greene, D. (2007). Evolving standards for informed consent: Is it time for an individualized and flexible approach? Professional Psychology: Research and Practice, 38 (2), 183-184.

${ }^{11}$ Larner, G. (2004). Family Therapy and the Politics of Evidence. Journal of Family Therapy. 26 (1): 17-39.

${ }^{12}$ Grant, J., \& Luxford, Y. (2009). Video: A decolonising strategy for intercultural communication in child and family health within ethnographic research. International Journal of Multiple Research Approaches, 3, 218-232

${ }^{13}$ Lee, A. (2007). Commentary: videotape recording of clinical encounters. Infant Observation, 10 (1), 87-88.

${ }^{14}$ Carroll, K., ledema, R., \& Kerridge, R. (2008). Reshaping ICU ward round practices using video-reflexive ethnography. Qualitative Health Research, 18 (3), 380-390

${ }^{15}$ Roy-Chowdhury, S. (2006). How is the therapeutic relationship talked into being? Journal of Family Therapy, 28, 153-174.

${ }^{16}$ Speer, S. \& Hutchby, I. (2003). From ethics to analytics: aspects of participants' orientations to the presence and relevance of recording devices. Sociology. 37 (2), 315-337.

${ }^{17}$ Beauchamp, T. (2007). The 'four principles approach' to healthcare ethics. In R. Ashcroft, A. Dawson, H. Draper and J. McMillan. Principles of Healthcare Ethics. West Sussex: John Wiley, 
${ }^{18}$ Roy-Chowdhury, S. (2003). Knowing the unknowable: what constitutes evidence in family therapy? Journal of Family Therapy, 25, 64-85

${ }^{19}$ Wooffitt, R. (2005). Conversation Analysis and Discourse Analysis: A Comparative and Critical Introduction. London: SAGE Publications.

${ }^{20}$ Hutchby, I. and Wooffitt, R. (1998). Conversation Analysis. Oxford: Blackwell Publishers.

${ }^{21}$ Atkinson, J. M. \& Heritage, J. (1999). Jefferson's transcript notation. In A. Jaworski, \& N.

Coupland, (Eds). The Discourse Reader. (pp: 158 - 166). London: Routledge.

${ }^{22}$ Beauchamp, T., and Childress, J. (2008). Principles of Biomedical Ethics. Sixth edition Oxford: Oxford University Press.

${ }^{23}$ Schiffrin, D. (1987). Discourse markers. Cambridge: Cambridge University Press.

${ }^{24}$ Heritage, J. (1984). A change-of-state token and aspects of its sequential placement. In J. M. Atkinson, \& J. Heritage, (Eds). Structures of social action: studies in conversation analysis. (pp: 299 - 345). Cambridge: Cambridge University Press.

${ }^{25}$ Bolden, G. (2006). Little words that matter: discourse markers "so" and "oh" and the doing of other-attentiveness in social interaction. Journal of Communication, 56, 661-688.

${ }^{26}$ Robinson, J. (2004). The sequential organisation of 'explicit' apologies in naturally occurring English. Research on Language and Social Interaction, 37, (3), 291-330.

${ }^{27}$ Barnett, J. (2007). Seeking an understanding of informed consent. Professional Psychology: Research and Practice, 38 (2), 179-182.

${ }^{28}$ Hewitt, J. (2007). Ethical component of researcher researched relationships in qualitative interviewing. Qualitative Health Research, 17, 1149 - 1159.

${ }^{29}$ Hofmann, B. (2009). Broadening consent- and diluting ethics? Journal of Medical Ethics, $35,125-129$. 
${ }^{30}$ Deming, N., Fryer-Edwards, K., Dudzinski, D., Starks, H., Culver, J., Hopley, E., Robins, L., \& Burke, W., (2007). Incorporating principles and practical wisdom in research ethics education: a preliminary study. Academic Medicine, 82 (1), 18 -23

${ }^{31}$ Haggerty, G., and Hilsenroth, M. (2011). The use of video in psychotherapy supervision. British Journal of Psychotherapy, 27 (2), 193-210

32 Themessl-Huber, M., Humphris, G., Dowell, J., Macgillivray, S., Rushmer, R., and Williams, B. (2008). Audio-visual recording of patient-GP consultations for research purposes: A literature review on recruiting rates and strategies. Patient Education and Counseling, 71 (2), 157-168. 